\title{
The adjusted index of location: Some methodological considerations
}

\author{
P. D. McCORMACK \\ Carleton University, Ottawa, Ontario K1S 5B6, Canada
}

\begin{abstract}
Adjusted index of location (AIL) scores may be employed in order to correct for the effects of response bias on location memory. These scores cannot be calculated, however, for subjects exhibiting very low false alarm rates. There are also difficulties associated with the analyses of collections of scores that contain large negative AIL values. Remedies for both of these situations are suggested and two experiments are reported, the results of which indicate that distortions in the data produced by these adjustments are minimal.
\end{abstract}

Hintzman and Block (1971) gave their subjects a serial list of 50 words and then required them to provide their best guess regarding the 10th of the list in which each word had been presented. In a situation such as this, subjects might be expected to sort the same number of words into each of the categories. This does not appear to be the case, however, since there is a strong response bias to avoid early and late categories (Hintzman, Block, \& Summers, 1973; McCormack, 1981, 1982; Toglia \& Kimble, 1976). This bias leads to a flattening of the slope of the function relating mean judged value and serial position. The bias presents a particularly serious problem when it is more pronounced for one group of subjects than for another, since spurious interactions are sometimes observed (McCormack, 1981).

One way of correcting for bias would be to calculate the probability that a word belongs in Category $i$ conditionalized on the event that it is judged to be in Category i (Hintzman et al., 1973). Another correction, and one that also provides credit for approximate accuracy in locating items, is given by the adjusted index of location (AIL) measure (Toglia \& Kimble, 1976). This procedure has the additional advantage of being applicable to the scores of individual subjects and, hence, of being amenable to statistical analysis. The two studies reported in this paper have arisen out of certain methodological considerations associated with AIL scores.

The AIL score provides a measure of memory for list location derivable from category sorting data for items presented previously (targets) as well as for items being presented for the first time (distractors). Adjustments

This study was funded by the Natural Sciences and Engineering Research Council of Canada (Grant A 7498), the Pickering Endowment Fund, and Carleton University Grant-in-Aid of Research GR5. The author wishes to acknowledge the assistance of Nancy Carboni in testing the subjects of Experiment 1 and of Susan McCullough in testing those of Experiment 2. Requests for reprints should be sent to P. D. McCormack, Department of Psychology, Carleton University, Colonel by Drive, Ottawa, Ontario K1S 5B6, Canada. for response bias are based on the way in which subjects assign distractors to the categories, that is, on their false alarm responding. Credit for less than perfect location accuracy is achieved by scoring correct target locations as zero and giving other responses values corresponding to the number of positions by which they deviate from the correct location. The AIL score, which has an upper bound of unity with chance performance indicated by scores in the vicinity of zero, may readily be calculated for groups of subjects from hit and false alarm input/output location matrices.

Statistical analyses, however, are based on the AIL scores of individual subjects. It is at this level of analysis that methodological problems arise. What should be done, for example, with the data of a subject who exhibits no false alarms and, therefore, for whom no measure of response bias can be obtained? How should one deal with negative AIL scores and the large variances associated with them? Certain adjustments will be performed and these will be considered as providing satisfactory solutions to these problems if it can be demonstrated that the conclusions reached on the basis of statistical analyses of the adjusted data correspond to those that would be arrived at following certain manipulations whose effects are known. The purpose of the two experiments reported here was to see if such a demonstration could be achieved.

\section{EXPERIMENT 1}

\section{Method}

The manipulation of major interest in this first experiment was the degree to which subjects are willing to sort items as targets. This was achieved by instructing one group to adopt a high sorting criterion and another to adopt a low criterion. Since this manipulation should not affect location memory accuracy, the AIL functions for the two groups of subjects were expected to be congruent.

Subjects and Materials. The subjects were 72 students of introductory psychology. The materials were 96 nouns whose mean imagery, concreteness, and meaningfulness values were as follows (with standard deviations reported in parentheses): 6.37 (.39), $6.72(.44)$, and $6.83(.81)$. 
Procedure. The 96 nouns were divided at random for each subject into two sets of 48 , the members of one set serving as targets and those of the other as distractors. The subjects were told that they would be tested for their ability to remember the locations of words in a serial list. The 48 targets were presented individually on cards, and in a different random order for each subject, at a 5-sec rate. These were then shown again, along with the 48 distractors, in a new random order. The subjects were instructed to give their best guess, while proceeding at their own pace, regarding the eighth of the list in which they had seen each target item. They achieved this by sorting the cards face down into piles labeled $1-8$, placing any "new" items in a discard pile. They were told that ideal performance would involve the assignment of targets having Input Serial Positions 1-6 to Category 1, 7-12 to Category 2, and so on. Half the subjects were assigned at random to a high-criterion group (Group HC) and were told to place a word in one of the eight piles only if they were absolutely certain that it had been seen previously. They were informed that there would be a penalty of 10 points for assigning a word that had not been seen, as well as a bonus of 5 points for each correct assignment. The remaining 36 subjects served as members of a low-criterion group (Group LC), who were told to always assign a word to a category unless they were absolutely sure that they had not seen it before. There was a bonus of 10 points for each word correctly assigned and a penalty of 5 points for each word incorrectly placed.

\section{Results}

The mean number of items sorted by Group LC was 48.11; that for Group HC was 37.67. The difference between these means was reliable $[t(70)=4.66$, $\mathrm{p}<.001$ ], indicating that the instructions had their predicted effect on sorting performance. The effects of the instructions were also evident in the recognition memory data. An examination of where the two groups set their criterion cutoffs indicated that subjects of Group HC exhibited more conservative behavior than did those of Group LC, the $\beta$ values for the two groups being 3.18 and 1.00 , respectively $(\mathrm{z}=8.07, \mathrm{p}<.001)$.

The number of distractors sorted by all subjects into Categories $1-8$ were $18,61,76,75,57,51,23$, and 12 , the modal categories being 3 and 4 for both groups. In order to estimate sorting bias, as well as to compute AIL scores for any given subject, two of the eight categories must have false alarm entries. This was not the case for 22 of the 36 subjects in Group HC, 10 of whom emitted no false alarms and 12 only one. Nor was it the case for 7 of the 36 subjects of Group LC, 4 of whom had no false alarms and 3 only one. In these cases, false alarms were inserted: When two were necessary, one was assigned to Category 3 and the other to Category 4 ; when only one insertion was necessary, Categories 3 and 4 were alternated across those subjects having only one false alarm. One other adjustment was performed on the data of individual subjects. Since AIL scores have an upper bound of 1.00 , it seemed reasonable to disallow any negative values greater than -1.00 . This adjustment has the effect of minimizing variance and was performed on the data of 24 subjects in Group HC and 16 in Group LC. In most cases, only one of the eight AIL scores for each subject needed adjustment.

In spite of the fact that more corrections of both kinds were necessary for the data of Group HC than for those of Group LC, there were still no significant differences in location-memory accuracy between the two groups of subjects, in the form either of a groups main effect $[F(1,68)<1]$ or of a Groups by Locations interaction $[F(7,476)<1]$. Only the input eighths main effect was statistically dependable $[F(7,476)=40.00$, $\mathrm{p}<.001]$, the usual primacy and recency effects being observed (McCormack, 1981, 1982; Toglia \& Kimble, 1976). The actual mean AIL scores for Groups HC and LC were .0856 and .0857 , respectively. This, of course, is what would be expected, given that the adjustments did not distort the data, since manipulations of sortingcriterion differences should not affect location-memory accuracy.

\section{EXPERIMENT 2}

\section{Method}

The manipulation of primary interest in the second experiment was the degree to which the subjects are exposed to targets during the study portion of the experimental session. One group received one study trial, whereas another received three. This manipulation was expected to affect location-memory accuracy, the AIL values for the latter group being significantly greater than those for the former group of subjects.

Subjects and Materials. The subjects were 50 students of introductory psychology. The materials were 144 nouns taken from Cluster 8 of the Toglia and Battig (1978) norms, 48 of these serving as targets and 96 as distractors for each subject. Their mean imagery, concreteness and meaningfulness values were as follows (with standard deviations reported in parentheses): $5.82(.55), 5.88(.31)$, and $4.79(.51)$.

Procedure. The procedure was essentially that of Experiment 1 , and the instructions were those given to Group LC of the first experiment, with the exception that the penalty for each word incorrectly placed was 1 point instead of 5 . Half of the subjects were given one trial (Group 1T) in which the 48 targets were presented at a 5 -sec rate, and the remaining subjects were given three trials (Group 3T). The targets were then shown again for both groups of subjects, intermingled with the 96 distractors, during which time they made their judgments regarding the eighths of the list in which they had seen each target item. As in the first experiment, different random orders of words were employed across subjects during both the study and test phases of the task.

\section{Results}

The trials manipulation had its expected effect on recognition memory performance, the $\mathrm{d}^{\prime}$ values for Groups $3 \mathrm{~T}$ and $1 \mathrm{~T}$ being 3.46 and 2.10 , respectively $(\mathrm{z}=14.11, \mathrm{p}<.001)$. The number of false alarms in each of the eight categories for all subjects was 31,53 , $60,62,68,50,59$, and 39 , the modal categories being 4 and 5 . Insertions of false alarms were necessary for 17 of the 25 subjects of Group 3T, in spite of the fact that they were given low-criterion instructions and had twice as many distractors as targets, both of these measures having been designed to maximize the false alarm emission rate. There were two false alarms inserted for five of these subjects, one into Category 4 and the other in to Category 5 , and one in either Category 4 or 5 for each of the remaining 12 subjects. For those subjects of Group 1T, two false alarms were inserted in one instance and one in another. Adjustments to negative AIL scores 
that exceeded -1.00 were needed, at least once, for six of the subjects of Group 1T and five of those of Group 3T. These two classes of transformations did not appear, however, to affect the expected outcome of the experiment. An analysis of the AIL data of individual subjects indicated a reliable main effect of groups $[F(1,48)=15.73, p<.001]$, those subjects with three serial learning trials showing better location memory than those with only one. These findings are consistent with those of Toglia and Kimble (1976), who report location-memory differences for one trial vs. two, but only for the first input eighth. The input eighths main effect was, of course, significant $[F(7,336)=20.13$, $\mathrm{p}<.001$, but the Groups by Input Eighths interaction was not $[F(7,336)=1.73, p>.10]$.

When the false alarm data for the two groups of subjects were examined separately, it was discovered that the modal category values were different, these being 4 and 5 for Group 1T and 2 and 7 for Group 3T. The individual AIL data for the subjects of Group 3T were, therefore, adjusted accordingly. The outcome of this analysis was identical to that of the earlier one. The main effect of groups was even larger than before $[F(1,48)=49.23, p<.001]$, the $F$ for interaction was less than 1 , and the input eighths main effect was, of course, once more significant $[F(7,336)=10.00$, $\mathrm{p}<.001]$.

\section{DISCUSSION}

The results of the two experiments reported here indicate that the adjustments did not produce any serious distortions in the data, since the conclusions reached from analyses of adjusted scores correspond to those that would have been predicted from the nature of the two sets of manipulations, there being no reliable differences between Groups HC and LC in Experiment 1, whereas there were significant differences between Groups 1T and $3 \mathrm{~T}$ in Experiment 2. It is, therefore, recommended that these adjustments be employed in future studies of location memory.
The reader might wish to have some estimate of the relative frequency of occurrence, in other experimental settings, of the two types of problems encountered here. Two investigations by McCormack are available for this purpose. In the first of these (McCormack, 1981), the task was identical to that employed in the two studies reported here and the subjects were 33 young and 33 elderly female adults. Eleven subjects required false alarm adjustments, and 25 had upper limits imposed on negative AIL scores. In the second investigation (McCormack, 1982), memory for spatial location was studied, again with young and elderly female adults serving as subjects. There were 176 subjects in all, 44 of these requiring false alarm adjustments and 24 needing negative AIL score corrections. These adjustments were not reported at the time and certainly should have been. In the light of the outcome of the present experiments, the failure to report the adjustments is not as serious as it otherwise might have been. In the only other available investigation employing the AIL transformation (Toglia \& Kimble, 1976), no mention whatever is made of procedures employed in the calculation of AIL scores for subjects exhibiting low false alarm emission rates.

\section{REFERENCES}

Hintzman, D. L., \& Block, R. A. Repetition and memory: Evidence for a multiple trace hypothesis. Journal of Experimental Psychology, 1971, 88, 297-306.

Hintzman, D. L., Block, R. A., \& Summers, J. J. Contextual associations and memory for serial position. Journal of Experimental Psychology, 1973, 97, 220-229.

McCormack, P. D. Temporal coding by young and elderly adults: A test of the Hasher-Zacks model. Developmental Psychology, 1981, 17, 509-515.

McCormaCK, P. D. Coding of spatial information by young and elderly adults. Journal of Gerontology, 1982, 37, 80-86.

Toglia, M. P., \& Battig, W. F. Handbook of semantic word norms. Hillsdale, N.J: Erlbaum, 1978.

Toglia, M. P., \& Kimble, G. A. Recall and use of serial position information. Journal of Experimental Psychology: Human Learning and Memory, 1976, 2, 431-445.

(Received for publication March 15, 1982.) 